\title{
Dexmedetomidine attenuates hyperalgesia induced by brachial plexus root avulsion by restoring the GLT- 1 function via PKA signaling
}

Xiaoying $\mathrm{Xu}$

Sun Yat-sen University

\section{Zhiheng Ren}

Sun Yat-sen University

\section{Ying Tang}

Southern University of Science and Technology

Prince L. M. Zilundu

Sun Yat-sen University

\section{Yingying Zhou}

Sun Yat-sen University

\section{Wenfu Li}

Sun Yat-sen University

Jiawei Hou

Sun Yat-sen University

\section{Yixin Fu}

Sun Yat-sen University

Yunlin Mai

Sun Yat-sen University

Yu Huang

Sun Yat-sen University

\section{Zaara Liaquats}

Sun Yat-sen University

\section{Xiao Cheng}

Guangdong Provincial Hospital of Traditional Chinese Medicine Jiang-Hong Ye

The State University of New Jersey, New Jersey Medical School

\section{Li-Hua Zhou}

Southern University of Science and Technology

Rao Fu ( $\square$ furao5@mail.sysu.edu.cn )

Southern University of Science and Technology 


\section{Research Article}

Keywords: BPRA, GLT-1, Dexmedetomidine, Pain, Neuroinflammation

Posted Date: March 16th, 2022

DOI: https://doi.org/10.21203/rs.3.rs-1400623/v2

License: (c) (i) This work is licensed under a Creative Commons Attribution 4.0 International License. Read Full License 


\section{Abstract}

Background: Chronic neuropathic pain often occurs with unclear mechanisms after brachial plexus root avulsion (BPRA) injuries. Emerging evidence suggests that the maladaptation of spinal glial glutamate transporter GLT-1 causes extracellular glutamate accumulation, contributing to central sensitization of chronic pain. Dexmedetomidine (DMET), an a2-adrenergic receptor (a2AR) agonist, widely used in the clinic as a sedative and analgesic drug, has been shown to inhibit glial activation. This study assessed DMET effects on BPRA induced pain and the possible involvement of GLT-1 regulation.

Methods: The right C8 and T1 roots were avulsed to establish a lower trunk BPRA injury rat model and LPS-induced activation of rat primary cultured astrocytes. Then we used the molecular and behavioral assay combined with pharmacological manipulation to test the hypothesis that DMET attenuates the pain and neuroinflammation through restoring the GLT-1 function via PKA signaling.

Results: The mechanical allodynia and thermal hyperalgesia appeared and reached the peak at 1-day post-injury (dpi) and persisted for at least $28 \mathrm{dpi}$. Notably, BPRA enhanced phosphorylated PKA levels, reduced GLT-1 expression, and caused an imbalance between anti- and proinflammatory cytokines in the affected spinal segments. Acute systemic or local DMET administration, at the un-sedative doses, demonstrated an analgesic effect. Moreover, a 3-days intrathecal DMET treatment ameliorated hyperalgesia and allodynia of BPRA injured rats by attenuating PKA phosphorylation, IL-1 $\beta$, and IL-6, while restoring the levels of GLT-1, IL-4, and IL-10 in the spinal cord. Significantly, intrathecal administration of the selective PKA inhibitor H89 mimicked, but the PKA activator 8-Br-cAMP blocked DMET's effects.

Conclusion: Overall, these results suggest that PKA inactivation mediates DMET's analgesic effect for the pain induced by BPRA injury through the recovery of GLT-1 function.

\section{Background}

Brachial plexus injuries usually cause sensorimotor function impairment and chronic pain symptoms [13]. While it is well documented that among the various types of lesions, the preganglionic lesions, the brachial plexus root avulsions (BPRA) were reported to have more frequently occurred in neuropathic pain $[4,5]$. Although quantities of studies contributed to the understanding of the cellular and molecular mechanisms involved in the development and maintenance of pain [6-8], there is still much to be known about the mechanism of neuropathic pain associated with avulsion injury, particularly when the lower trunk are affected $[2,4]$.

It has been well accepted that the hyper-excitability of dorsal horn pain projection neurons ("central sensitization") is a primary substrate for neuropathic pain [9-14]. The precisely balanced control of glutamate release and uptake has recently merged as essential regulating targets in treating neuropathic pain, including those related to spinal nerve injury [15-19]. In the tripartite synaptic architecture, 
astrocytic processes minimize glutamate overflow by rapidly uptaking glutamate, thereby permitting efficient synaptic signaling[20, 21].

A family of glutamate transporters clears the extracellular and synaptic glutamate. Specifically, the glial excitatory amino acid transporter 2 (EAAT2/GLT-1), situated in the astrocytic processes, functions as a scavenger to prevent glutamate spillover into the extrasynaptic space[22-24] through rapidly uptaking glutamate [25, 26]. GLT-1 also regulates glutamatergic synaptic transmission [27-30], thereby modulating neuronal activity [31]. Moreover, the GLT-1 function was linked with the pathogenesis of neuropathic pain $[18,32]$ associated with peripheral nerve injury $[33,34]$ and spinal cord injury $[16,35-37]$.

In addition, the central sensitization is also driven by neuroinflammation in either the peripheral or central nervous system [11]. We recently reported that BPRA elicited severe neuroinflammation, characterized by microglial and astrocyte activation. This leads to the release of proinflammatory cytokines and chemokines, like CXCL1, ICAM1, IP10, MCP-5, MIP1- $a$ and CD93 in the injured spinal segments [38]. Interestingly, the GLT-1 function has also been closely linked with glial cell activation and cytokines released during chronic pain $[39,40]$, but whether GLT-1 involves the spinal cord neuroinflammation caused by BPI remains unknown.

The dexmedetomidine (DMET), an agonist of alpha2-adrenoreceptors (a2-AR), has been widely used clinically for sedation and anesthesia. Recently, DMET has been extensively reported to exert the potent neuroprotective effect of alleviating neuronal apoptosis [41, 42], mediated by suppressing reactive gliosis and excitotoxicity [43]. Moreover, DMET was reported to effectively diminish chronic pain driven by neuroinflammation [44], suggesting the potential role of a2-AR in regulating glial activation at the spinal level, but the molecular mechanism undetermined/unspecified $[45,46]$.

Considering the close relationship of reactive gliosis and aberrant hyperactivity of spinal sensory neurons affected by avulsion injury, the current study was sought to investigate whether DMET could relieve the pain caused by BPRA injury at the lower trunk and whether the glutamate transporter involved the perspective mechanism. In this study, we firstly evaluated the feature of chronic pain caused by BPRA. Then we used in vivo and in vitro studies based on the molecular and behavioral assay combined with pharmacological manipulation to test the hypothesis that DMET attenuates the pain and neuroinflammation through restoring the GLT-1 function via PKA signaling.

\section{Materials And Methods}

\section{Animals}

Experiments were conducted on male adult Sprague-Dawley rats (8-week-old at the experiments). The number of animal use permits is SYSU-IACUC-2018-000241. Surgical and animal care procedures were carried out under the provisions outlined in the National Health and Medical Research Council animal ethics and ARRIVE guidelines. All procedures were performed with the approval of the Animal Care and Utilization Committee of Sun Yat-sen University. Rats were housed in ventilated Plexiglas cages in a 
climate-controlled room $\left(20^{\circ} \mathrm{C}\right)$ under a 12-hour light/dark cycle (lights off at 8:00 P.M). Animals were allowed to acclimatize to the housing conditions and handling before the start of each experiment. Food and water were available ad libitum or otherwise indicated.

\section{C8 and T1 spinal roots avulsion}

The microsurgery procedure for the BPRA injury was minorly modified based on what we previously described $[47,48]$. Briefly, the anesthesia was induced in rats with ketamine/xylazine $(80 / 20 \mathrm{mg} / \mathrm{kg}$, i.p.) and maintained with $1 \%$ isoflurane. Each animal was laid out in the supine position, following the retraction of the paravertebral muscles. The right side $\mathrm{C} 6$ and $\mathrm{C} 7$ laminectomy were performed under a surgical microscope, and then the dura mater was opened. After identifying the C8 and T1 segments of the spinal cord for the BPRA group, their spinal roots were selectively avulsed one by one. The proximal residual rootlets and the distal parts of the $\mathrm{C} 8$ and $\mathrm{T} 1$ avulsed roots were cut away to ensure that spinal MNs would not regrow axons into the damaged nerves in the avulsed rats. All the avulsed distal parts of roots were cut away, and the success of the avulsion model was confirmed under the microscope for the animals in the sham control group. The lower trunk was exposed and isolated but not avulsed. Finally, the muscle, fascia, and skin were sutured successively in layers in all animals. The body temperature of rats remained at $36.5 \pm 0.5^{\circ} \mathrm{C}$ throughout the procedure. The pain behaviors were evaluated $24 \mathrm{~h}$ after surgery.

\section{Western blot assay}

The western blot assay was performed as described [49]. Briefly, at 7 days post-injury (dpi) followed BPRA injury or $2 \mathrm{~h}$ after pharmacological behavioral tests, the rats were perfused transcardially with icecold saline under deep anesthesia with sodium pentobarbital (50 mg/kg, i.p.) and dorsal part of the C8-T1 spinal segments was collected under a microscope, then stored at $-80^{\circ} \mathrm{C}$ until processing. After that, the lysis and protein extraction of spinal cord tissues was performed using the RIPA lysate buffer (P0013, Beyotime). The concentration of the extracted protein was determined by the BCA Protein Assay Kit (Cat No: 23225, Pierce Biotechnology). The $40 \mu \mathrm{g}$ of protein extracts from each sample were denatured and subjected to $4-15 \%$ Tris $-\mathrm{HCl}$ precast Mini Protean ${ }^{\circledR}$ TGX ${ }^{\mathrm{TM}}$ gels (BioRad) for electrophoresis. Proteinseparated PVDF membranes (Bio-Rad, Philadelphia, PA) were blocked with 5\% non-fat milk (Bio-Rad) in TBST (in mM, 24 Tris, $\mathrm{pH} \mathrm{7.4,137} \mathrm{NaCl,} 2.7 \mathrm{KCl}$, and 0.05\% Tween 20) for $1 \mathrm{~h}$ at room temperature and incubated with antibodies against GLT-1 antibody (1:1000, ab41621, Abcam), a2-AR (1:1000, ab 85570, Abcam), GLAST (1:1000, ab 41751, Abcam), EAAC1 (1:1000, ab 124802, Abcam), total PKA (1:1000, ab216572, Abcam), phosphorylated PKA Thr197 (1:1000, ab75991, Abcam) and GAPDH (1:3000, G8795, Sigma Aldrich, St. Louis, MO) overnight at $4{ }^{\circ} \mathrm{C}$. After washing with TBST, the membranes were incubated for $2 \mathrm{~h}$ with goat anti-rabbit or mouse IgG-HRP conjugated antibody (1:2500, Jackson Immuno-Research, West Grove, PA) at room temperature and developed with ECL solutions (PerkinElmer, Waltham, MA). All protein signals were analyzed by scanning densitometry using Image Lab software (Bio-Rad) and normalized to GAPDH.

\section{Immunofluorescence and cell counting}


The rats were anesthetized with sodium pentobarbital (50 mg/kg, i.p.) and perfused with $4 \%$ PFA. Then, the cervical spinal cord was dissected under the surgical microscope. Notably, the C8-T1 spinal segments were defined as the region between the uppermost and the lowermost rootlets of the contralateral C7-C8 spinal nerves. The tissue was post-fixed with 4\% PFA and dehydrated by overnight immersion in $30 \%$ $(\mathrm{v} / \mathrm{v})$ sucrose in phosphate buffer solution at $4{ }^{\circ} \mathrm{C}$, followed by a frozen section at $30 \mu \mathrm{m}$.

Next, every third section of the spinal cord sections was chosen for the standard immunostaining as we previously described [47]. The sections were rinsed three times in PBS and then treated with $0.3 \%$ Triton $\mathrm{X}-100$ and $0.1 \%$ bovine serum albumin (BSA) in PBS at room temperature for $30 \mathrm{~min}$. These sections were further incubated overnight at $4{ }^{\circ} \mathrm{C}$. The following primary antibodies were used: mouse anti-NeuN (1:500, ab104224, Abcam, UK), rabbit anti-GFAP (1:500, 80788S, CST, USA), rabbit anti-IBA-1 (1:500, 01919741, Wako Bioproducts, USA), rabbit anti-pCREB S133 (1:200, ab32096, Abcam, UK). Then, following washing three times with PBS, the sections were incubated with respective secondary antibodies. The following antibodies were used: Goat anti-Mouse IgG $(\mathrm{H}+\mathrm{L})$, Cross-Adsorbed Secondary Antibody, Alexa Fluor 488 (1:1500, A32723, Invitrogen, USA), Goat anti-Rabbit IgG $(\mathrm{H}+\mathrm{L})$ Cross-Adsorbed Secondary Antibody, Alexa Fluor 555 (1:1000, A32732, Invitrogen, USA). Incubation was $2 \mathrm{~h}$ at room temperature in the dark after rinsing the sections three times with PBS. In addition, the staining without primary or secondary antibodies served as negative controls. The immunofluorescence (IF) was evaluated under a fluorescence microscope (Nikon Eclipse 90i, Japan). The average fluorescence intensity (AFI) and the number of GFAP- or IBA-1-immunoreactive (IR) cells in each rat for 8-10 sections were evaluated with the Nikon N.I.S. software package under $10 \times$ magnification.

\section{Behavioral analysis}

Mechanical and thermal tests were carried out before BPRA injury and at different time points after surgery. Each behavioral test was carried out at $1 \mathrm{~h}$ intervals. The paw withdrawal thresholds (PWT) in rats in response to mechanical stimuli (calibrated von Frey filaments) were measured as we previously described [50-53]. Briefly, the animal was placed in an individual Plexiglas chamber on an elevated mesh screen. For rats, calibrated von Frey filaments (Stoelting Co., Wood Dale, IL, USA) in log increments of force $(0.69,1.20,2.04,3.63,5.50,8.51,15.14$, and $26 \mathrm{~g})$ were used to stimulate the plantar surface of the rats' left and right hind paws. The 3.63-g stimulus was used first. If a negative response occurred, then the next larger von Frey hair was applied, if a positive response was seen, then the next smaller von Frey hair was applied. The application was terminated when (i) a negative response was seen with the $26-\mathrm{g}$ stimulation or (ii) three stimuli were used after the first positive response. Based on a formula provided by Dixon [54], the PWT was calculated by converting the pattern of positive and negative responses to a $50 \%$ threshold value.

Paw withdrawal latencies (PWL) to thermal stimulation were then examined with a Model 336 Analgesia Meter (IITC Inc. Life Science Instruments. Woodland Hills, CA), as described previously [50, 53]. In brief, the animal was placed in an individual Plexiglas chamber on a glass plate. A beam of light through a hole in the lightbox of Model 336 Analgesia Meter and thence through the glass plate was used to 
stimulate the middle of the plantar surface of each hind paw. The light beam was automatically turned off when the animal withdrew its foot. The PWL was defined as light beam onset to paw withdrawal. Each test was repeated five times at 5-min intervals for the paw on each side. The cut-off time of $20 \mathrm{~s}$ was set up to avoid tissue damage.

The open-field test was utilized to screen the sub-anesthetic dose of DMET by assessing its effect on the animal locomotor activity as we previously described [55]. The apparatus consisted of four fixed walls around a plastic square floor $(43.2 \times 43.2 \times 30.5 \mathrm{~cm})$. The illumination was $30-50$ lux. Each animal was gently placed in the open field center, and its behavior was recorded. The total distance traveled of animals was measured by software (SOF-811, Med Associates Inc, St. Albans, VT).

\section{Chemicals and application}

All common salts were purchased from Sigma Aldrich (USA), including the DMET (1-100 $\mu \mathrm{g} / \mathrm{kg}$, i.p. $1 \mu \mathrm{g} / 10 \mu \mathrm{l}$, i.t.), selective a2AR antagonist BRL-44408 (100 $\mu \mathrm{g} / 10 \mu \mathrm{l}$, i.t.), a2CR antagonist JP-1302 $(0.1 \mu \mathrm{g} / 10 \mu \mathrm{l}$, i.t.), PKA inhibitor $\mathrm{H} 89$ dihydrochloride ( $\mathrm{H} 89,5 \mu \mathrm{g} / 10 \mu \mathrm{l}$, i.t.), and PKA activator 8-Bromo-cAMP (8-Br-CAMP, $300 \mu \mathrm{M} / 10 \mu$ l, i.t.). The doses of these chemicals were selected based on our pilot study and previous reports [56]. All chemicals were dissolved in a mixture of sterile aCSF and suspended in $10 \%$ Dimethyl Sulfoxide (DMSO), 40\% PEG 300, 5\%Tween-80, 45\% Saline, solution following pilot work that confirmed this concentration by allowing passage of high concentrations of this hydrophobic drug through the fine-gauged microinjectors.

\section{Cell lines and cell treatments}

We studied the influence of DMET/PKA signaling on proinflammatory stimulation of human astrocyte 1321N1 cells (Cat No: 86030402, Sigma-Aldrich) with lipopolysaccharides (LPS). The cells were maintained in DMEM (Gibco, Invitrogen, USA) supplemented with $10 \%$ fetal bovine serum in a $5 \% \mathrm{CO}_{2}$ incubator at $37^{\circ} \mathrm{C}$ for 2 weeks. The cells were then seeded in 6-well plates and subjected to the following 30 min pretreatment before stimulation with $100 \mathrm{ng} / \mathrm{ml}$ LPS for 24 hours. 1) DMET (1 $\mu \mathrm{M})$ alone, 2) DMET combined with a2A receptor antagonist BRL-44408 $(5 \mu \mathrm{M}), 3)$ DMET with a2C receptor antagonist JP-1302(1 $\mu \mathrm{M})$, 4) DMET with PKA activator 8-Br-cAMP $(250 \mu \mathrm{M})$, 5) selective PKA inhibitor $\mathrm{H} 89(20 \mu \mathrm{M})$. The doses of the above chemicals and treating time were chosen based on previous studies [57, 58]. After that, the cells were harvested for histology, western blotting, and ELISA measurement.

\section{Enzyme-linked immunosorbent assay (ELISA)}

Tissue levels of proinflammatory cytokines such as tumor necrosis factor- $\alpha$ (TNF- $\alpha$ ), interleukin-1 $\beta$ (IL$1 \beta), I L-6, I L-18, I L-33$, as well as anti-inflammatory cytokines such as IL-4 and IL-10 in the spinal dorsal horn were quantified via enzyme-linked immunosorbent assay (ELISA) according to the protocol provided by the manufacturer (USCN Life Science, Wuhan, China). As we previously reported, the detailed procedure was modified [59]. The right dorsal horn of the C8-T1 spinal segment was dissected on ice and 
then immediately transferred into the liquid nitrogen. Tissue samples were homogenized in a lysis buffer containing protease and phosphatase inhibitors. Homogenates were centrifuged at $4{ }^{\circ} \mathrm{C}$ for $10 \mathrm{~min}$ at 2,500 rpm, and the supernatants were collected. The Bradford method was used to quantify the concentration of each cytokine. Using an optical density (OD) of $450 \mathrm{~nm}$, standard curves of the analyzed cytokines were generated, and the concentrations of inflammatory factors were calculated.

\section{Statistical Analysis}

All data were expressed as a mean \pm SEM (standard mean error). Animal sample sizes chosen to ensure adequate statistical power were determined based on our preliminary studies. Animals were randomly assigned to different studies. Investigators were blinded to group allocations in the pharmacological behavioral experiments. Before the analysis, all data were checked for normality and homogeneity of variances by using Sigma-Aldrich, Plot 12.5. For histology staining and protein expression, the relative fluorescence intensity and protein expression were analyzed using the two-tailed unpaired student's t-test between two groups or one-way ANOVA followed by Turkey's for multiple comparisons. The data of the behavioral tests, including pain and open field tests, were analyzed using a one- or two-way repeatedmeasures ANOVA, followed by Bonferroni or LSD. Posthoc tests. Statistical significance was declared at $p<0.05$.

\section{Results}

\section{Brachial plexus roots avulsion (BPRA) injury increases mechanical and thermal nociceptive sensitivity, as well as enhances the astrocyte activation and neuronal activity}

Forty rats were included in this experiment to determine the effect of brachial plexus roots avulsion (BPRA) injury on the nociceptive sensitivity. Firstly, these rats were assessed by analyzing the paw withdrawal threshold (PWT) and latency (PWL) to the mechanical and thermal stimuli before surgery, and no significant difference was detected in the pain sensitivity of affected forepaws among these animals (mechanical pain: $F_{234}=2.144, p \otimes 0.1327$, thermal pain: $F_{234}=2.486, p \otimes 0.0983$, Fig. 1 ). Next, they were randomly divided into three subgroups to receive C8-T1 roots avulsion (BPRA, $n=13$ ) or sham surgery (Sham, $n=12$ ), and the rest of them served as normal control counterparts (Normal, $n=12$ ). Three rats were excluded since the failure of nerve roots was completed avulsed. A two-way RM ANOVA revealed a significant group $\times$ time interaction effect on PWT $\left(F_{16272}=84.82, p \llbracket 0.0001\right)$, and PWL $\left(F_{16} 272=165.8, p\right.$ $₫ 0.0001$ ) of affected forelimb during 1-28 day post-injury (dpi). Post-hoc analyses found that the BPRA rats demonstrated robust and long-lasting mechanical allodynia and heat hyperalgesia on the forepaws, reflected by the greatly reduced PWL and PWT in the nociception test as compared to either sham or normal control animals (all $p<0.0001$ ). Particularly, it is noted that these pain hypersensitivities occurred and reached a peak 1 day after avulsion surgery and persisted for at least 28 dpi (Fig.1).

Also, we observed that BPRA injury significantly induced gliosis by histological evidence that the enhanced GFAP immunoreactivity and the number of NeuN/pCREB labeled cells observed in the dorsal 
horn of the affected spinal segments, as compared to either normal or sham rats (Fig.1). In addition, since no significance was found between normal and sham-treated rats, the two groups were pooled together in the following experiments.

\section{Acute DMET treatment alleviates the hyperalgesia caused by BPRA}

To determine whether avulsion-caused hyperalgesia involves spinal a2-AR, we measured the nociception of BPRA rats by systemic or local pharmacologically manipulated a2-AR. Firstly, we examined whether systemic administration of DMET could alleviate the cervical root's avulsion-induced pain. We screened the sub-anesthetic dose of DMET by assessing its effect on the animal locomotor activity in the open field test. DMET at 30 to 100 , but not 5 to $20(\mu \mathrm{g} / \mathrm{kg}$, dissolved in saline, IP) significantly reduced the total distance traveled in 60 min test session (One-way ANOVA $F_{863}=70.99, p<0.0001$ ). Particularly, rats demonstrated an approximately 20-30 min transient hypnotic phase after administration with DMET at the dose of 50 (5 of 8 rats) and 100 (8 of 8 rats) during the test session (Supplementary Fig.1). Next, the effect of DMET ( 5 to $20 \mu \mathrm{g} / \mathrm{kg}$ ) was evaluated in an independent group of avulsion and sham rats at 1 dpi. The avulsion injured but non-sham rats showed mechanical allodynia and thermal hyperalgesia at 30 min before drug infusion, consistent with the abovementioned (Fig.1) and severing as the baseline of pharmacological nociception test. The two-way RM ANOVA revealed a significant group (Sham vs BPRA) $\times$ Treatment (saline vs DEMT) interaction on mechanical $\left(F_{348}=29.44, p<0.0001\right)$ and thermal pain $\left(F_{3} 48\right.$ $=4.034, p=0.012$ ) of affected forepaws. DMET at doses 5,10 , and $20 \mu \mathrm{g} / \mathrm{kg}$ significantly alleviate the PWT of rats when challenged with mechanical stimuli, compared to saline, in a clear dose-dependent manner. Moreover, we also observed that DMET elevated the PWT of sham-treated rats. While, DMET at $20 \mu \mathrm{g} / \mathrm{kg}$ partly reduces the PWL of avulsion injured, but not as compared to saline during thermal pain test (Fig.2 a,b).

In addition, the analgesic effect of systemic DMET administration was also confirmed by the intrathecal DMET application. As shown in Fig.2, we observed that intra-thecal DMET $(1 \mu \mathrm{g})$ significantly elevated the reduced the PWT and PWL caused by BPRA. More importantly, this analgesic effect was abolished by coinjection of a2A receptor antagonist BRL-44408, but not by the selective a2C receptor antagonist JP-1302, suggesting the spinal a2A receptor activation is required for DMET analgesic effect (Fig.2 c,d).

\section{Chronic DMET treatment alleviates the hyperalgesia and restores the spinal GLT-1 expression of BPRA injured Rats}

In furtherance of our studies, we determined the effect of chronic DMET administration on the nociception of BPRA injured rats. The behavior results demonstrated that DMET (i.t. $1 \mu \mathrm{g}$ ) treatment for 3 days, immediately following the injury, significantly reduced the BPRA induced paw flinches in mechanical and thermal pain assessment during the 7 days observation period (Fig.3 PWT, treatment $\times$ time interaction $F_{521}=6.42, p<0.001$, PWL, $F_{521}=6.42, p<0.001$ treatmentxtime interaction). Considering aberrant spinal glutamate metabolism-associated pain behavior and a2A receptor[60], we measured how avulsion injury and DMET treatment alter GLT-1 and a2A receptor-related signaling. As 
shown in Fig.3, avulsion injury significantly decreased GLT-1, a2AR level compared to sham surgery but enhanced the PKA phosphorylation expression level. All of the above changes were rectified through the 3-day DMET local treatment (all $p<0.05$ ). We did not observe that DMET administration significantly alters these protein levels in sham-operated rats (all $p>0.05$ ). Moreover, although EAAT2 (GLAST) and EAAT3 (EAAC1) also involve the glutamate reuptake [61], we reported that neither avulsion injury nor DMET treatment significantly altered their level in the affected spinal segment. (EAAT2, unpaired t-test, $t$ $(9)=10.73, p=0.0041$, EAAT3, unpaired $t$-test, $t(9)=10.73, p=0.0041)$. The above results suggest that the impaired GLT-1 might serve as an effector in response to PKA phosphorylation and a2A receptor activation, contributing to the pain associated with avulsion injury.

\section{PKA inhibition is required for DMET analgesic effect and spinal GLT-1 level restoration in BPRA rats}

Next, we set up the pharmacological behavior test to further determine the role of PKA in the DMET analgesic effect and cytokine level in BPRA rats. The BPRA injured rats were divided into four subgroups to examine the effect on mechanical and thermal nociception when intrathecal administration of vehicle, DMET, DMET combined with PKA activator 8-Bromo-cAMP, or PKA selective inhibitor H89 alone, respectively. The animals that received sham operations served as control groups. All the chemicals were injected daily into animal rats for 3 days, at 30 min before the onset of the behavior assessment.

The two-way RM ANOVA revealed a significant treatment $x$ time on both PWT $\left(F_{271}=3.691, p=0.041\right)$ and PWL $\left(F_{271}=4.368, p=0.025\right)$. Post-hoc analysis revealed that, as compared to Sham or BPRA+vehicle group, DMET treatment ( $2 \mu$ g i.t., 3 days) significantly reduced the hyperalgesia and allodynia during 7 days observation period. However, the analgesic effect was diminished when 8-BrcAMP was in presence. Moreover, the DMET analgesic effect was mimicked by $\mathrm{H} 89$ (all $p<0.05$, Fig. 4 $a, b)$, suggesting the PKA inactivation might be triggered by DMET involving the pain control processing.

All animals were sacrificed for spinal protein and cytokines assays immediately when the last behavior terminated to assess how PKA function manipulation affects the GLT-1 expression and inflammation level in the affected spinal cord. The WB assay showed that pharmacological activation of PKA resulted in an upregulated PKA phosphorylation level, which also mitigated the GLT-1 restoration induced by DMET. In contrast, pharmacological inhibition of PKA decreased phosphorylated PKA level, accompanied with restored GLT-1 expression in BPRA rats (all $p<0.05$, Fig. 4c).

In addition, chronic DMET treatment demonstrated an anti-inflammatory effect by suppressing the elevated TNF-a, IL-1 $\beta$, and IL- 6 levels, restoring the reduced IL-4 and IL-10 levels in injured spinal segments caused by avulsion injury. However, these effects were blocked by the presence of PKA activator 8-Br-cAMP and mimicked by using PKA inhibitor H89 alone (all $p<0.05$, Fig. 4d).

DMET restored the GLT-1 level and suppressed inflammation in LPS induced astrocytes by inhibiting PKA signaling 
Next, to confirm the underlying mechanisms of DMET in BPRA injury, we further examined the effect of DMET in LPS-induced astrocytes cell lines. As shown in Fig.5, results from fluorescence histological evidence showed that astrocytes subjected to $24 \mathrm{~h}$ lipopolysaccharide (LPS, $100 \mathrm{ng} / \mathrm{ml}$ ) exposure significantly enhanced the GFAP level, reflected by increased GAPF positive cells number, suggesting astrocyte activation. LPS exposure also significantly activated PKA signaling, reflected by the enhanced phosphorylation level in staining and WB assay.

What's more, the above changes were significantly suppressed by treating cells with $1 \mu \mathrm{M}$ DMET for 24 hours. However, the a2A receptor antagonist BRL-44408 $(5 \mu \mathrm{M})$ but not the selective a2C receptor antagonist JP-1302 $(1 \mu \mathrm{M})$ impaired the DMET effect (all $p<0.05$,), suggesting that the a2A receptor activation is required for its suppression on astrocyte activation induced by LPS (Fig.5a-c).

Finally, DMET treatment efficiently restored the diminished GLT-1 expression in astrocytes under LPS exposure, which was impeded by adding 8-Br-cAMP $(250 \mu \mathrm{M})$ in the culture medium. Plus, the reduced GLT-1 level of LPS-exposed cells was recovered when the cells were pretreated with the PKA inhibitor H89 $(20 \mu \mathrm{M})$ alone (Fig. $5 \mathrm{~d}-\mathrm{h})$. The levels of inflammatory factors were also determined by using ELISA assays. Compared to LPS stimulated astrocytes, the stimulated cells treated either with DMET or PKA inhibitor $\mathrm{H} 89$ substantially lower the elevated levels of IL-1 $1 \beta, \mathrm{IL}-6$, and TNF-a, while significantly improving IL-10 levels. However, the DMET's anti-inflammatory effect was impaired when the PKA activator 8-BrcAMP was mixed in the treatment (Supplementary Figure 2, all $p<0.05$ ), suggesting the PKA played a critical role in participating in the anti-inflammatory effect of DMET.

\section{Discussion}

We report here that BPRA injured rats displayed allodynia and hypersensitivity to mechanical and thermal stimuli, accompanied by impaired GLT-1 function, elevated astrocyte activation, and increased inflammation in affected spinal segments. Dexmedetomidine, administered acute systemically or chronic locally using the non-sedative dose, shows a potent analgesic and anti-inflammatory effect. Additionally, we proposed that a2AR/PKA/GLT-1 signaling of spinal astrocytes might be involved in the aforementioned biological events.

BPRA induces a robust, fast-onset, and long-lasting pain on the ipsilateral forepaws. This pain occurs immediately after the injury and lasts for at least three months in rat models, whether partial or complete roots avulsion [5, 62-64]. Here we identified that the pain started from $1 \mathrm{dpi}$ and was sustained for 28 days during the observation on both ipsilateral and contralateral forepaws in a trunk brachial lower plexus rat model, which is consistent with previous observations reports [62-64]. After three days of administration of DMET from 7 days post-injury, both the mechanical allodynia and the thermal hyperalgesia were alleviated, suggesting the potent analgesic effect of DMET, particularly with intrathecal usage.

Besides the pain behavior, we observed that the expression of PCREB in the affected spinal dorsal horn increased robustly 7 days after BPRA. The permanent neuronal hyperactivity observed in the spinal cord 
posterior horn in peripheral neuropathy cases may be due to the preservation and persistence of the connection between the sensory ganglia and the CNS neurons [65]. Considering the phosphorylation form of CREB (pCREB) is pivotal for both inducing and maintaining the long-term neuronal plasticity (LTP) in spinal dorsal horn neurons and accepted as an essential biomarker for central sensitization [12, 66-68], we thence proposed that avulsion injury-induced pain is partly caused by neuronal activation, which is consistent with the view in previous studies $[66,69-71]$.

In addition, the glia cell over-activation in the spinal cord dorsal horn is closely related to peripheral nerve injury-induced pain [64, 72-74]. We reported here the GFAP was highly activated in the injured spinal dorsal horn at the avulsed section 7 days after injury, which is also supported by the evidence presented in the previous report [72].

One of the primary routes of glutamate clearance in the spinal cord is through GLT-1. Moreover, the glutamate transporter, GLT-1, is highly expressed in astrocytes and modulates extracellular glutamate levels to maintain the CNS's homeostasis in normal physiological conditions [75]. Therefore, downregulation of spinal GLT-1 expression following elevated levels of extracellular glutamate often serves as a common mechanism of neuropathic pain and underlies the development and maintenance of central sensitization [76]. The dysfunction of GLT-1 may be attributed to the genetic, epigenetic, transcriptional, or translational changes in astrocytes $[76,77]$. When the astrocytes are activated upon exposure to nerve injury, they undergo a set of morphological, transcriptional, and functional changes that transform them into different cells, with acquired, lost, or altered properties and functions [78]. In our study, the injured spinal dorsal horn GLT-1 expression decreased 7 days after BPRA, and this decrease was partly reversed by DMET, suggesting that DMET might reduce BPRA-induced pain by recovering GLT1 function via gliosis inhibition. Our results are supported by plenty of previous studies. Like the spinal administration of Riluzole, a positive glutamate transporter activity regulator, effectively attenuated neuropathic pain [32], whereas the inhibition of glutamate transporters increased spinal extracellular glutamate levels, leading to the development of spontaneous pain $[79,80]$. More recently, a vector that selectively increases GLT1 expression in astrocytes when microinjected into the superficial spinal dorsal horn was developed and used to show that GLT1 overexpression in astrocytes reverses established neuropathic pain following spinal cord injury [81].

Although we agree that the downregulation of glutamate transporters often precedes or occurs simultaneously with the development of hypersensitivity to thermal or tactile stimuli in various models of chronic pain, we only found the level of GLT-1, but neither EAAT2 nor EAAT3, decreased. The probable explanation for such findings is attributable to the downregulation onset time of GLT-1 being earlier than other transporters in response to injury [82]. Future study is required to investigate how these transporters change with a much broader time window followed injury.

One limitation of the current study is that we did not address the mechanism underlying GLT-1 downregulated by the avulsion injury. However, we proposed that post-translational regulation, including sumoylation, palmitoylation, nitrosylation, ubiquitination, and subcellular localization, might be involved 
[83]. In addition, we reported here that chronic DMET treatment restored the reduced GLT-1 expression in both affected spinal cord and cultured astrocytes, suggesting a regulatory role of DMET on glutamate transporters. This result is consistent with a previous study that DMET upregulates GLT-1 levels by $\mathrm{PI3K} / \mathrm{AKT}$-dependent pathway in an ischemia stroke rodent model [84]. Meanwhile, we also noticed a controversial result that showed that DMET downregulated GLT-1 mRNA levels in rat astrocytic cell lines [85]. We considered that the mRNA and protein levels measured in the two studies might have been the cause for inconsistency.

Given that activation of both alpha-2A or $2 \mathrm{C}$ in the spinal cord, especially in the lamina II, directly reduces pain transmission [58], we subsequently further assured whether the DMET induced analgesia was restored GLT-1 expression is via the alpha-2A receptor. In doing such, we set up the pharmacological behavioral test and western blot to assess the action of DMET in the presence of alpha-2A or $2 \mathrm{C}$ receptor antagonists. It's noted that the DMET's analgesic and restored GLT expression effect was significantly blocked by a2A receptor antagonist BRL44408, but not by JP-1302, suggesting the selective role of alpha$2 \mathrm{~A}$ receptor participation in the above molecular events. These observations are supported by the welldocumented evidence showing that the analgesic effect modulation of a2-adrenoceptor agonists is via the a2A subtype $[86,87]$. Therefore, we proposed that the reduced GLT-1 expression likely contributes to the onset of pain behavior, serving as one of the modulatory targets of DMET treatment.

The a2A receptor belongs to the $\mathrm{Gi} /$ o protein-coupled receptor family, and its activation leads to a cascade of events, such as inactivation of adenylate cyclase (AC) activity and cAMP/PKA pathway [88]. We tested the hypothesis that a2A activation restores the GLT-1 function by inhibiting PKA signaling in astrocytes. Using the selective PKA agonist and antagonist, combined with in vivo and in vitro studies, we observed the DMET restored GLT-1 protein level was blocked when the PKA activation, especially the PKA inhibitor, mimicked the effect of a2A activation. Based on the observation, avulsion injury or LPS stimulated inflammation enhanced the expression of phosphorylated PKA and reduced GLT-1 expression. These phenomena infer that PKA inhibition is required for GLT-1 upregulation by a2A. Moreover, PKA signaling in the astrocyte might physiologically suppress the GLT-1 protein expression.

\section{Conclusion}

In conclusion, we demonstrated that the neuropathic pain associated with brachial plexus downregulates GLT-1 protein expression at the astrocyte level. The selective agonist a2A DMET displays an analgesic and anti-inflammatory effect by restoring the reduced GLT-1 expression through PKA signaling inhibition. Our findings provide new insight into the mechanisms involved in the downregulation of GLT-1 in chronic pain models.

\section{Abbreviations}

a2AR: a2-adrenergic receptor, AC: Adenylate cyclase, AFI: average fluorescence intensity, BPRA: Brachial plexus root avulsion, DMET: Dexmedetomidine, DPI: Day post-injury, EAAT2: Excitatory amino acid 
transporter 2, ELISA: Enzyme-linked immunosorbent assay, GFAP: Glial fibrillary acidic protein, GLT-1: Glutamate transporter, IL: Interleukin, LPS: Lipopolysaccharide, OD: Optical density, PWL: Paw withdrawal latency, PWT: Paw withdrawal threshold, TNF-a: Tumor necrosis factor-a,

\section{Declarations}

\section{Ethics approval and consent to participate}

All procedures were performed according to the Chinese National Health and Medical Research Council animal ethics guidelines and with the approval of the Sun Yat-sen University Animal Experimentation Ethics Committee (SYSU-IACUC-2018-000241).

\section{Consent for publication}

Not applicable

\section{Availability of data and materials}

All data generated or analyzed during this study are included in this published article.

\section{Competing interests}

The authors declare that they have no known competing financial interests or personal relationships that could have influenced the work reported in this paper.

\section{Funding}

This work is generously supported by the National Natural Science Foundation of China (82071496), Natural Science Foundation of Guangdong (2021A1515010463), and the Young Teacher Foundation of Sun Yat-sen University (59000-18841219) to Rao Fu.

\section{Authors' contributions}

$R F, L H Z$, JHY and $X C$ were responsible for conceiving and designing the study, XYX, ZHR, YT, PLMZ, WFL participated in animal surgery and established the animal model, YYZ, YH, JWH, YXF, YLM, ZL pharmacological animal pain testing, and histological experiments. ZHR, YYZ, and YT conducted the glial cell culture and pharmacological treatment, western-blot, and related ELISA experiments. RF conducted the data analysis and interpretation. RF and JHY composed and edited the manuscript. RF, LHZ, and XC supervised the project.

\section{Acknowledgements}

Not applicable 


\section{References}

1. Bruxelle J, Travers V, Thiebaut JB: Occurrence and treatment of pain after brachial plexus injury. Clin Orthop Relat Res 1988:87-95.

2. Anand $P$, Birch R: Restoration of sensory function and lack of long-term chronic pain syndromes after brachial plexus injury in human neonates. Brain 2002, 125:113-122.

3. Berman JS, Birch R, Anand P: Pain following human brachial plexus injury with spinal cord root avulsion and the effect of surgery. Pain 1998, 75:199-207.

4. Parry WCB: Pain in avulsion lesions of the brachial plexus. Pain 1980, 9:41-53.

5. Teixeira MJ, da Paz MG, Bina MT, Santos SN, Raicher I, Galhardoni R, Fernandes DT, Yeng LT, Baptista AF, de Andrade DC: Neuropathic pain after brachial plexus avulsion-central and peripheral mechanisms. BMC Neurol 2015, 15:73.

6. Ji RR, Xu ZZ, Gao YJ: Emerging targets in neuroinflammation-driven chronic pain. Nat Rev Drug Discov 2014, 13:533-548.

7. Davis KD, Aghaeepour N, Ahn AH, Angst MS, Borsook D, Brenton A, Burczynski ME, Crean C, Edwards $R$, Gaudilliere $B$, et al: Discovery and validation of biomarkers to aid the development of safe and effective pain therapeutics: challenges and opportunities. Nat Rev Neurol 2020, 16:381-400.

8. Truini A, Garcia-Larrea L, Cruccu G: Reappraising neuropathic pain in humans-how symptoms help disclose mechanisms. Nat Rev Neurol 2013, 9:572-582.

9. Gwak YS, Hulsebosch CE: Neuronal hyperexcitability: a substrate for central neuropathic pain after spinal cord injury. Curr Pain Headache Rep 2011, 15:215-222.

10. Campbell JN, Meyer RA: Mechanisms of neuropathic pain. Neuron 2006, 52:77-92.

11. Ji RR, Nackley A, Huh Y, Terrando N, Maixner W: Neuroinflammation and Central Sensitization in Chronic and Widespread Pain. Anesthesiology 2018, 129:343-366.

12. Latremoliere A, Woolf $\mathrm{CJ}$ : Central sensitization: a generator of pain hypersensitivity by central neural plasticity. J Pain 2009, 10:895-926.

13. Woolf CJ: Evidence for a central component of post-injury pain hypersensitivity. Nature 1983, 306:686-688.

14. Woolf CJ, Mannion RJ: Neuropathic pain: aetiology, symptoms, mechanisms, and management. Lancet 1999, 353:1959-1964.

15. Putatunda R, Hala TJ, Chin J, Lepore AC: Chronic at-level thermal hyperalgesia following rat cervical contusion spinal cord injury is accompanied by neuronal and astrocyte activation and loss of the astrocyte glutamate transporter, GLT1, in superficial dorsal horn. Brain Res 2014, 1581:64-79.

16. Falnikar A, Hala TJ, Poulsen DJ, Lepore AC: GLT1 overexpression reverses established neuropathic pain-related behavior and attenuates chronic dorsal horn neuron activation following cervical spinal cord injury. Glia 2016, 64:396-406. 
17. Wang XM, Gu P, Saligan L, ladarola M, Wong SSC, Ti LK, Cheung CW: Dysregulation of EAAT2 and VGLUT2 Spinal Glutamate Transports via Histone Deacetylase 2 (HDAC2) Contributes to Paclitaxelinduced Painful Neuropathy. Mol Cancer Ther 2020, 19:2196-2209.

18. Napier IA, Mohammadi SA, Christie MJ: Glutamate transporter dysfunction associated with nerve injury-induced pain in mice. $J$ Neurophysiol 2012, 107:649-657.

19. Yoshizumi M, Eisenach JC, Hayashida K: Valproate prevents dysregulation of spinal glutamate and reduces the development of hypersensitivity in rats after peripheral nerve injury. J Pain 2013, 14:1485-1491.

20. Rusakov DA, Zheng $K$, Henneberger $C$ : Astrocytes as regulators of synaptic function: a quest for the Ca2+ master key. Neuroscientist 2011, 17:513-523.

21. Perea G, Navarrete M, Araque A: Tripartite synapses: astrocytes process and control synaptic information. Trends Neurosci 2009, 32:421-431.

22. Zhou Y, Danbolt NC: Glutamate as a neurotransmitter in the healthy brain. J Neural Transm (Vienna) 2014, 121:799-817.

23. Ginsberg SD, Martin LJ, Rothstein JD: Regional deafferentation down-regulates subtypes of glutamate transporter proteins. J Neurochem 1995, 65:2800-2803.

24. Mitani A, Tanaka K: Functional changes of glial glutamate transporter GLT-1 during ischemia: an in vivo study in the hippocampal CA1 of normal mice and mutant mice lacking GLT-1. J Neurosci 2003, 23:7176-7182.

25. Rothstein JD, Patel S, Regan MR, Haenggeli C, Huang YH, Bergles DE, Jin L, Dykes Hoberg M, Vidensky $S$, Chung DS, et al: Beta-lactam antibiotics offer neuroprotection by increasing glutamate transporter expression. Nature 2005, 433:73-77.

26. Vandenberg RJ, Ryan RM: Mechanisms of glutamate transport. Physiol Rev 2013, 93:1621-1657.

27. Ayers-Ringler JR, Jia YF, Qiu YY, Choi DS: Role of astrocytic glutamate transporter in alcohol use disorder. World J Psychiatry 2016, 6:31-42.

28. Murphy-Royal C, Dupuis JP, Varela JA, Panatier A, Pinson B, Baufreton J, Groc L, Oliet SH: Surface diffusion of astrocytic glutamate transporters shapes synaptic transmission. Nat Neurosci 2015, 18:219-226.

29. Trantham-Davidson H, LaLumiere RT, Reissner KJ, Kalivas PW, Knackstedt LA: Ceftriaxone normalizes nucleus accumbens synaptic transmission, glutamate transport, and export following cocaine self-administration and extinction training. J Neurosci 2012, 32:12406-12410.

30. Weng HR, Chen JH, Pan ZZ, Nie H: Glial glutamate transporter 1 regulates the spatial and temporal coding of glutamatergic synaptic transmission in spinal lamina II neurons. Neuroscience 2007, 149:898-907.

31. Cui W, Mizukami H, Yanagisawa M, Aida T, Nomura M, Isomura Y, Takayanagi R, Ozawa K, Tanaka K, Aizawa $\mathrm{H}$ : Glial dysfunction in the mouse habenula causes depressive-like behaviors and sleep disturbance. J Neurosci 2014, 34:16273-16285. 
32. Sung B, Lim G, Mao J: Altered expression and uptake activity of spinal glutamate transporters after nerve injury contribute to the pathogenesis of neuropathic pain in rats. $J$ Neurosci 2003, 23:28992910.

33. Dmitrieva N, Rodriguez-Malaver AJ, Perez J, Hernandez L: Differential release of neurotransmitters from superficial and deep layers of the dorsal horn in response to acute noxious stimulation and inflammation of the rat paw. Eur J Pain 2004, 8:245-252.

34. Pitcher MH, Ribeiro-da-Silva A, Coderre TJ: Effects of inflammation on the ultrastructural localization of spinal cord dorsal horn group I metabotropic glutamate receptors. J Comp Neuro/ 2007, 505:412423.

35. Maeda S, Kawamoto A, Yatani Y, Shirakawa H, Nakagawa T, Kaneko S: Gene transfer of GLT-1, a glial glutamate transporter, into the spinal cord by recombinant adenovirus attenuates inflammatory and neuropathic pain in rats. Mol Pain 2008, 4:65.

36. Nicholson KJ, Gilliland TM, Winkelstein BA: Upregulation of GLT-1 by treatment with ceftriaxone alleviates radicular pain by reducing spinal astrocyte activation and neuronal hyperexcitability. $J$ Neurosci Res 2014, 92:116-129.

37. Lin Y, Roman K, Foust KD, Kaspar BK, Bailey MT, Stephens RL: Glutamate Transporter GLT-1 Upregulation Attenuates Visceral Nociception and Hyperalgesia via Spinal Mechanisms Not Related to Anti-Inflammatory or Probiotic Effects. Pain Res Treat 2011, 2011:507029.

38. Zhong K, Li Y, Tang Y, Yu G, Zilundu PLM, Wang Y, Zhou Y, Xu X, Fu R, Zhou L: Cytokine profile and glial activation following brachial plexus roots avulsion injury in mice. $J$ Neuroimmunol 2021, 353:577517.

39. Tai $Y H$, Wang $Y H$, Wang JJ, Tao PL, Tung CS, Wong CS: Amitriptyline suppresses neuroinflammation and up-regulates glutamate transporters in morphine-tolerant rats. Pain 2006, 124:77-86.

40. Tilleux S, Hermans E: Neuroinflammation and regulation of glial glutamate uptake in neurological disorders. J Neurosci Res 2007, 85:2059-2070.

41. Degos V, Charpentier TL, Chhor V, Brissaud O, Lebon S, Schwendimann L, Bednareck N, Passemard S, Mantz J, Gressens P: Neuroprotective effects of dexmedetomidine against glutamate agonistinduced neuronal cell death are related to increased astrocyte brain-derived neurotrophic factor expression. Anesthesiology 2013, 118:1123-1132.

42. Wang $X$, Shan Y, Tang Z, Gao L, Liu H: Neuroprotective effects of dexmedetomidine against isoflurane-induced neuronal injury via glutamate regulation in neonatal rats. Drug Des Devel Ther 2019, 13:153-160.

43. Pan W, Lin L, Zhang N, Yuan F, Hua X, Wang Y, Mo L: Neuroprotective Effects of Dexmedetomidine Against Hypoxia-Induced Nervous System Injury are Related to Inhibition of NF-kappaB/COX-2 Pathways. Cell Mol Neurobiol 2016, 36:1179-1188.

44. Xu B, Zhang WS, Yang JL, Xu H, Deng XM, Zhang YQ: Dexmedetomidine blocks thermal hyperalgesia and spinal glial activation in rat model of monoarthritis. Acta Pharmacologica Sinica 2010, 31:523530. 
45. Xu B, Zhang WS, Yang JL, Lu N, Deng XM, Xu H, Zhang YQ: Evidence for suppression of spinal glial activation by dexmedetomidine in a rat model of monoarthritis. Clin Exp Pharmacol Physiol 2010, 37:e158-166.

46. Liu L, Ji F, Liang J, He H, Fu Y, Cao M: Inhibition by dexmedetomidine of the activation of spinal dorsal horn glias and the intracellular ERK signaling pathway induced by nerve injury. Brain Res 2012, 1427:1-9.

47. Tang Y, Fu R, Ling ZM, Liu LL, Yu GY, Li W, Fang XY, Zhu Z, Wu WT, Zhou LH: MiR-137-3p rescue motoneuron death by targeting calpain-2. Nitric Oxide-Biology and Chemistry 2018, 74:74-85.

48. Tang Y, Ling ZM, Fu R, Li YQ, Cheng X, Song FH, Luo HX, Zhou LH: Time-specific microRNA changes during spinal motoneuron degeneration in adult rats following unilateral brachial plexus root avulsion: ipsilateral vs. contralateral changes. BMC Neurosci 2014, 15:92.

49. Fu R, Tang Y, Li W, Ren Z, Li D, Zheng J, Zuo W, Chen X, Zuo QK, Tam KL, et al: Endocannabinoid signaling in the lateral habenula regulates pain and alcohol consumption. Transl Psychiatry 2021, 11:220.

50. Zhao JY, Liang L, Gu X, Li Z, Wu S, Sun L, Atianjoh FE, Feng J, Mo K, Jia S, et al: DNA methyltransferase DNMT3a contributes to neuropathic pain by repressing Kcna2 in primary afferent neurons. Nat Commun 2017, 8:14712.

51. Fu R, Gregor D, Peng Z, Li J, Bekker A, Ye J: Chronic intermittent voluntary alcohol drinking induces hyperalgesia in Sprague-Dawley rats. Int J Physiol Pathophysiol Pharmacol 2015, 7:136-144.

52. Gregor DM, Zuo W, Fu R, Bekker A, Ye JH: Elevation of Transient Receptor Potential Vanilloid 1 Function in the Lateral Habenula Mediates Aversive Behaviors in Alcohol-withdrawn Rats. Anesthesiology 2019, 130:592-608.

53. Li Z, Gu X, Sun L, Wu S, Liang L, Cao J, Lutz BM, Bekker A, Zhang W, Tao YX: Dorsal root ganglion myeloid zinc finger protein 1 contributes to neuropathic pain after peripheral nerve trauma. Pain 2015, 156:711-721.

54. Dixon WJ: Efficient analysis of experimental observations. Annu Rev Pharmacol Toxicol 1980, 20:441-462.

55. Fu R, Zuo W, Shiwalkar N, Mei Q, Fan Q, Chen X, Li J, Bekker A, Ye JH: Alcohol withdrawal drives depressive behaviors by activating neurons in the rostromedial tegmental nucleus. Neuropsychopharmacology 2019, 44:1464-1475.

56. Ma D, Hossain M, Rajakumaraswamy N, Arshad M, Sanders RD, Franks NP, Maze M:

Dexmedetomidine produces its neuroprotective effect via the alpha 2A-adrenoceptor subtype. Eur $J$ Pharmacol 2004, 502:87-97.

57. Liu H, Davis JR, Wu ZL, Faez Abdelgawad A: Dexmedetomidine Attenuates Lipopolysaccharide Induced MCP-1 Expression in Primary Astrocyte. Biomed Res Int 2017, 2017:6352159.

58. Ishii H, Kohno T, Yamakura T, Ikoma M, Baba H: Action of dexmedetomidine on the substantia gelatinosa neurons of the rat spinal cord. Eur J Neurosci 2008, 27:3182-3190. 
59. Mi J, Yang Y, Yao H, Huan Z, Xu C, Ren Z, Li W, Tang Y, Fu R, Ge X: Inhibition of heat shock protein family A member 8 attenuates spinal cord ischemia-reperfusion injury via astrocyte NFkappaB/NLRP3 inflammasome pathway : HSPA8 inhibition protects spinal ischemia-reperfusion injury. J Neuroinflammation 2021, 18:170.

60. Guo W, Imai S, Zou S, Yang J, Watanabe M, Wang J, Dubner R, Wei F, Ren K: Altered glial glutamate transporter expression in descending circuitry and the emergence of pain chronicity. Mol Pain 2019, 15:1744806918825044.

61. Gegelashvili G, Bjerrum OJ: High-affinity glutamate transporters in chronic pain: an emerging therapeutic target. J Neurochem 2014, 131:712-730.

62. Liu Y, Wang L, Meng C, Zhou Y, Lao J, Zhao X: A new model for the study of neuropathic pain after brachial plexus injury. Injury 2017, 48:253-261.

63. Wang L, Yuzhou L, Yingjie Z, Jie L, Xin Z: A new rat model of neuropathic pain: complete brachial plexus avulsion. Neurosci Lett 2015, 589:52-56.

64. Kobayashi T, Yamauchi K, Matsuura Y, Kuniyoshi K, Takahashi K, Ohtori S: The Effects of Generally Administered Anti-Nerve Growth Factor Receptor (p75NTR) Antibody on Pain-Related Behavior, Dorsal Root Ganglia, and Spinal Glia Activation in a Rat Model of Brachial Plexus Avulsion. $J$ Hand Surg Am 2015, 40:2017-2025.

65. Gold MS: Spinal nerve ligation: what to blame for the pain and why. Pain 2000, 84:117-120.

66. Song XS, Cao JL, Xu YB, He JH, Zhang LC, Zeng YM: Activation of ERK/CREB pathway in spinal cord contributes to chronic constrictive injury-induced neuropathic pain in rats. Acta Pharmacol Sin 2005, 26:789-798.

67. Wheeler DG, Barrett CF, Groth RD, Safa P, Tsien RW: CaMKII locally encodes L-type channel activity to signal to nuclear CREB in excitation-transcription coupling. J Cell Biol 2008, 183:849-863.

68. Xin WJ, Gong QJ, Xu JT, Yang HW, Zang Y, Zhang T, Li YY, Liu XG: Role of phosphorylation of ERK in induction and maintenance of LTP of the C-fiber evoked field potentials in spinal dorsal horn. $J$ Neurosci Res 2006, 84:934-943.

69. Liu S, Mi WL, Li Q, Zhang MT, Han P, Hu S, Mao-Ying QL, Wang YQ: Spinal IL-33/ST2 Signaling Contributes to Neuropathic Pain via Neuronal CaMKII-CREB and Astroglial JAK2-STAT3 Cascades in Mice. Anesthesiology 2015, 123:1154-1169.

70. Ma W, Quirion R: Increased phosphorylation of cyclic AMP response element-binding protein (CREB) in the superficial dorsal horn neurons following partial sciatic nerve ligation. Pain 2001, 93:295-301.

71. Liou JT, Liu FC, Hsin ST, Yang CY, Lui PW: Inhibition of the cyclic adenosine monophosphate pathway attenuates neuropathic pain and reduces phosphorylation of cyclic adenosine monophosphate response element-binding in the spinal cord after partial sciatic nerve ligation in rats. Anesth Analg 2007, 105:1830-1837, table of contents.

72. Hou AL, Xu WD: A model of neuropathic pain in brachial plexus avulsion injury and associated spinal glial cell activation. $J$ Pain Res 2018, 11:3171-3179. 
73. Shen J, Huo BB, Zheng MX, Hua XY, Shen H, Lu YC, Jiang DL, Shan CL, Xu JG: Evaluation of Neuropathic Pain in a Rat Model of Total Brachial Plexus Avulsion from Behavior to Brain Metabolism. Pain Physician 2019, 22:E215-e224.

74. Matsuura Y, Iwakura N, Ohtori S, Suzuki T, Kuniyoshi K, Murakami K, Hiwatari R, Hashimoto K, Okamoto S, Shibayama M, et al: The effect of Anti-NGF receptor (p75 Neurotrophin Receptor) antibodies on nociceptive behavior and activation of spinal microglia in the rat brachial plexus avulsion model. Spine (Phila Pa 1976) 2013, 38:E332-338.

75. Ji RR, Donnelly CR, Nedergaard M: Astrocytes in chronic pain and itch. Nat Rev Neurosci 2019, 20:667-685.

76. Ramos KM, Lewis MT, Morgan KN, Crysdale NY, Kroll JL, Taylor FR, Harrison JA, Sloane EM, Maier SF, Watkins LR: Spinal upregulation of glutamate transporter GLT-1 by ceftriaxone: therapeutic efficacy in a range of experimental nervous system disorders. Neuroscience 2010, 169:1888-1900.

77. Pajarillo E, Rizor A, Lee J, Aschner M, Lee E: The role of astrocytic glutamate transporters GLT-1 and GLAST in neurological disorders: Potential targets for neurotherapeutics. Neuropharmacology 2019, 161:107559.

78. Escartin C, Guillemaud O, Carrillo-de Sauvage MA: Questions and (some) answers on reactive astrocytes. Glia 2019, 67:2221-2247.

79. Liaw WJ, Stephens RL, Jr., Binns BC, Chu Y, Sepkuty JP, Johns RA, Rothstein JD, Tao YX: Spinal glutamate uptake is critical for maintaining normal sensory transmission in rat spinal cord. Pain 2005, 115:60-70.

80. Weng HR, Chen JH, Cata JP: Inhibition of glutamate uptake in the spinal cord induces hyperalgesia and increased responses of spinal dorsal horn neurons to peripheral afferent stimulation. Neuroscience 2006, 138:1351-1360.

81. Ji W, Jiang W, Li M, Li J, Li Z: miR-21 deficiency contributes to the impaired protective effects of obese rat mesenchymal stem cell-derived exosomes against spinal cord injury. Biochimie 2019, 167:171-178.

82. Tao YX, Gu J, Stephens RL, Jr.: Role of spinal cord glutamate transporter during normal sensory transmission and pathological pain states. Mol Pain 2005, 1:30.

83. Peterson AR, Binder DK: Post-translational Regulation of GLT-1 in Neurological Diseases and Its Potential as an Effective Therapeutic Target. Front Mol Neurosci 2019, 12:164.

84. Peng M, Ling X, Song R, Gao X, Liang Z, Fang F, Cang J: Upregulation of GLT-1 via PI3K/Akt Pathway Contributes to Neuroprotection Induced by Dexmedetomidine. Front Neuro/ 2019, 10:1041.

85. Kurita M, Matsuoka Y, Nakatsuka K, Ono D, Muto N, Kaku R, Morimatsu H: Norepinephrine-induced downregulation of GLT-1 mRNA in rat astrocytes. Biochem Biophys Res Commun 2018, 504:103-108.

86. Altman JD, Trendelenburg AU, MacMillan L, Bernstein D, Limbird L, Starke K, Kobilka BK, Hein L: Abnormal regulation of the sympathetic nervous system in alpha2A-adrenergic receptor knockout mice. Mol Pharmacol 1999, 56:154-161. 
87. Ma D, Rajakumaraswamy N, Maze M: alpha2-Adrenoceptor agonists: shedding light on neuroprotection? Br Med Bull 2004, 71:77-92.

88. Wu YY, Goldfien A, Roberts JM: Alpha adrenergic stimulation reduces cyclic adenosine 3',5'monophosphate generation in rabbit myometrium by two mechanisms.Biol Reprod 1988, 39:58-65.

\section{Figures}
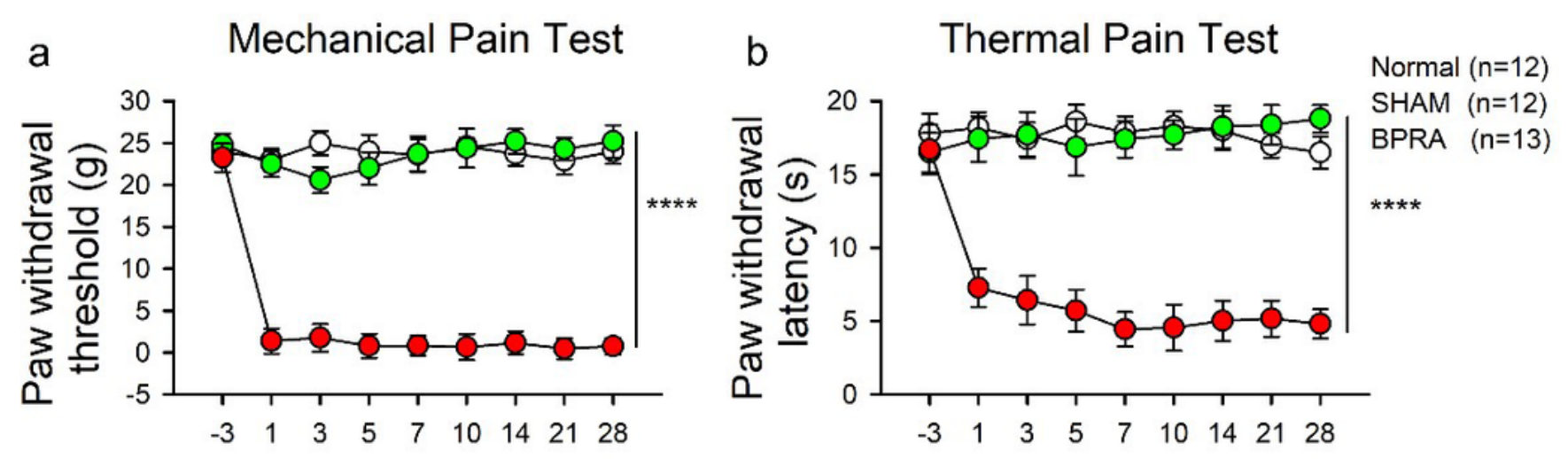

Days post-surgery

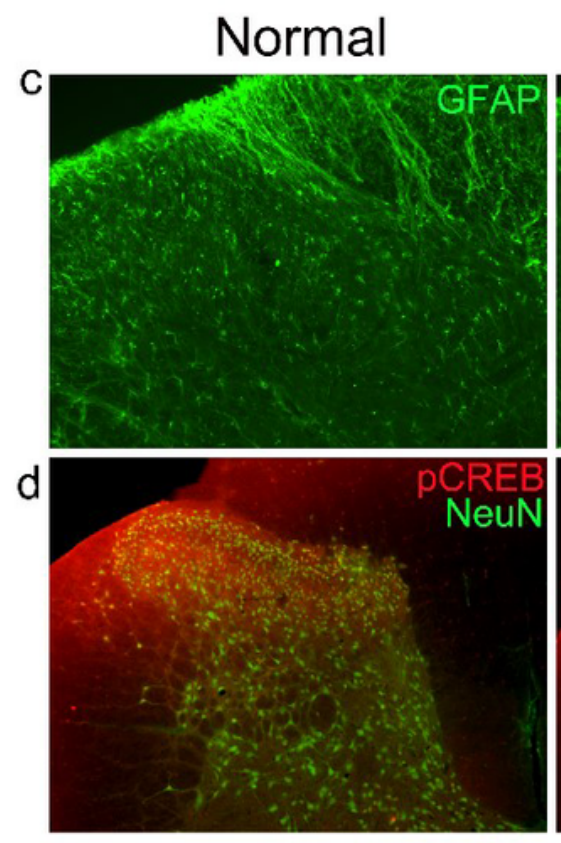

SHAM

\section{BPRA}
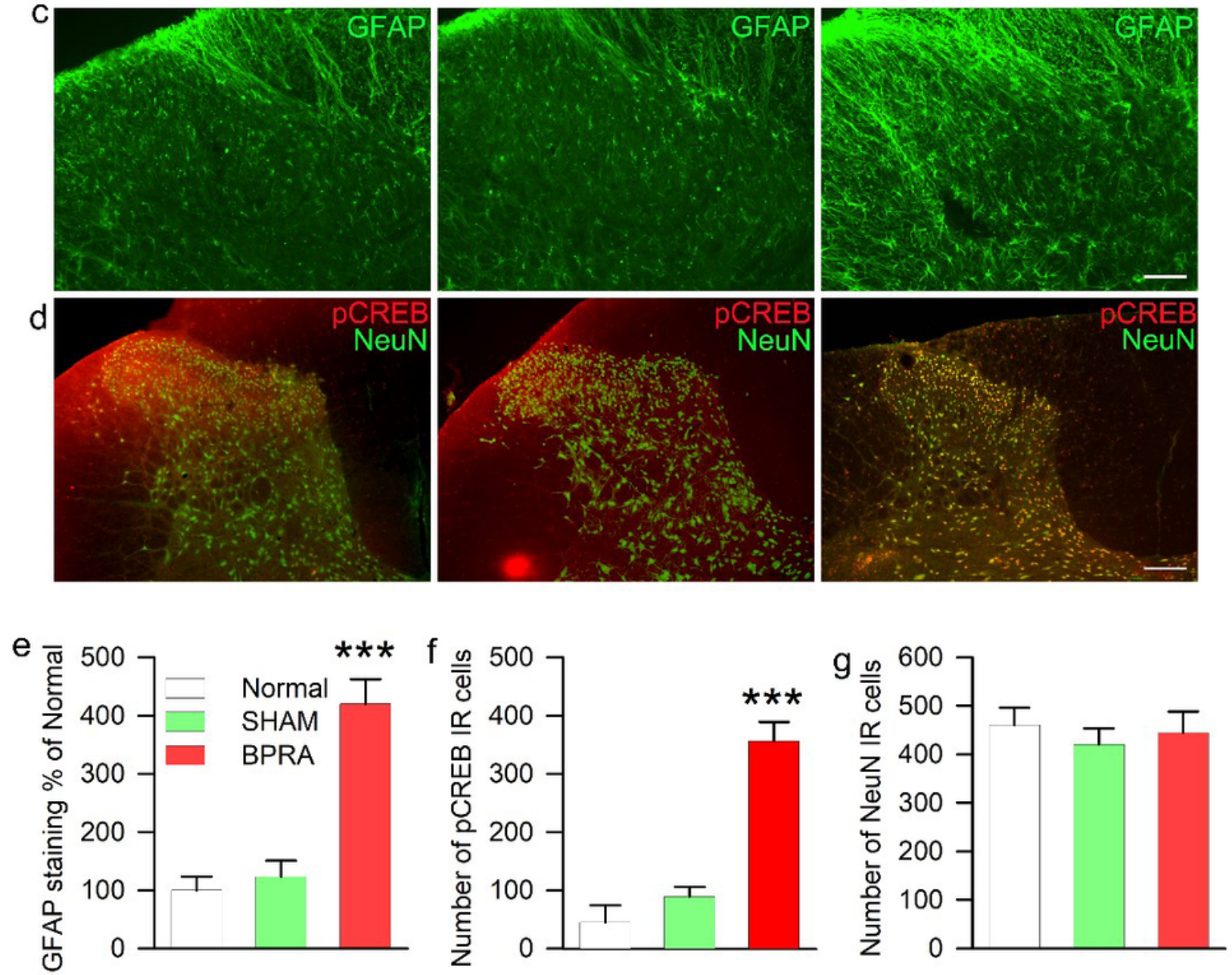


\section{Figure 1}

Spinal nerve roots avulsion elicits a rapid occurred and long-lasting pain and enhances the astrocyte activation and neuronal pCREB expression. Adult Sprague-Dawley male rats underwent unilateral C8-T1 brachial plexus roots avulsion injury (BPRA, $n=13$,) or sham surgery (Sham, $n=12$ ) were assessed by way of the paw withdrawal threshold (PWT) and paw withdrawal latency (PWL) in response to mechanical or noxious thermal stimuli during the baseline or period of 1-28 days post-injury (dpi). The aged-and bodyweight-matched naïve rats served as normal control (Normal, $n=12$ ). The $\mathbf{a}-\mathbf{b}$ summarizes the PWT and PWL of the above rats, showing that BPRA lesion causes both mechanical and thermal hyperalgesia of upper limbs of animals, ${ }^{* \star * \star} p<0.0001$ vs. Sham or Normal group, respectively, analyzed by two-way RM ANOVA. The lower panel depicts the representative micro image of GFPA(c), NeuN/pCREB (d) colocalization of the affected spinal dorsal horn, and the fluorescence intensity of immunoreactivity and immunoreactive (IR) cells number was summarized in panel $\mathbf{e g}$, ${ }^{* \star} p<0.001$ by one-way ANOVA. Scale bar $=100 \mu \mathrm{m}$ (panel c) or $200 \mu \mathrm{m}$ (panel d). 
Sysmetic DMET administration

a

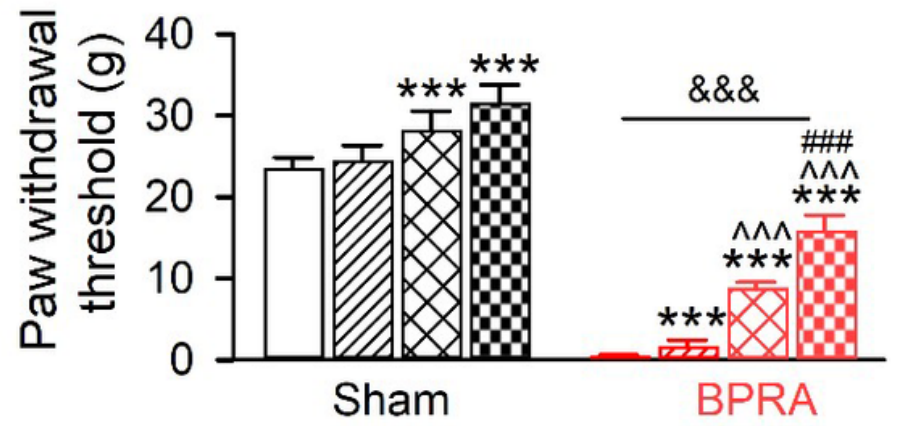

b

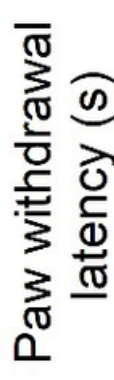

Intrathecal DMET administration

C

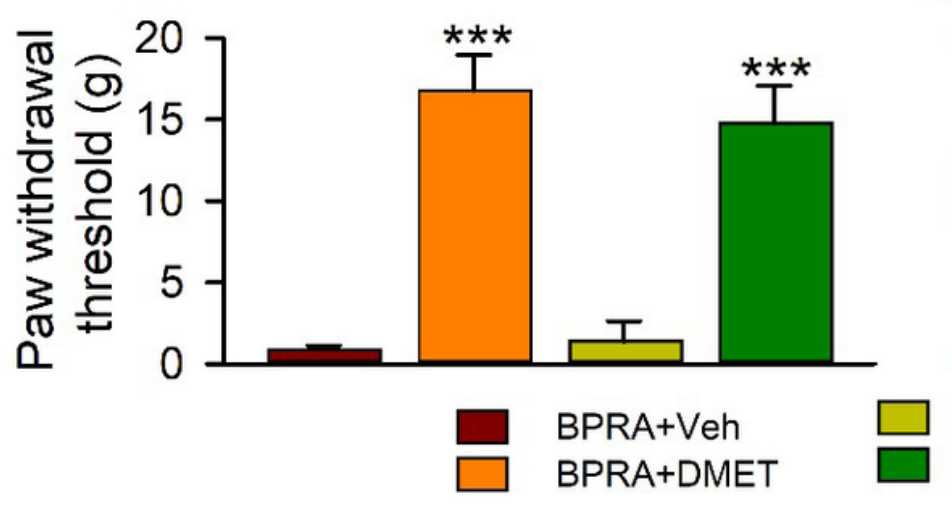

d

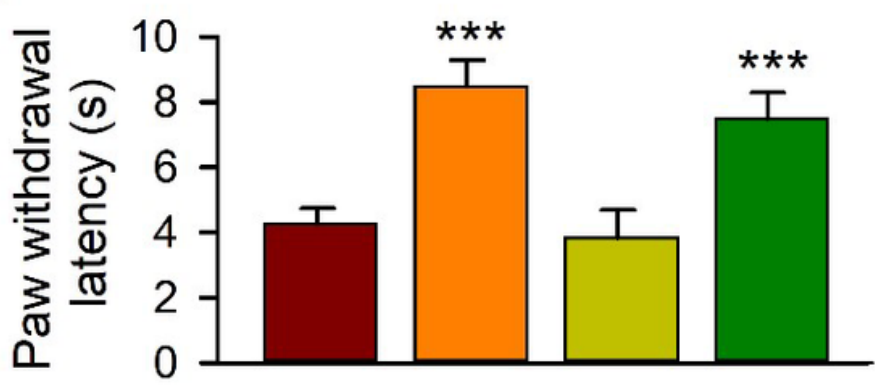

BPRA+DMET+BLR-44408

BPRA+DMET+JP-1302

Figure 2

Acute dexmedetomidine (DMET) administration has an analgesic effect on rats suffering from brachial plexus roots avulsionखBPRA囚injury through spinal alpha-2A receptor activation. Adult male SD rats that underwent C5-T1 BPRA injury or Sham lesion operation were randomly divided into systemic DMET treatment groups (5-10 $\mu \mathrm{g} / \mathrm{kg}$, i.p., $\mathrm{n}=10-13 /$ dose) and a control group of rats injected with the same volume saline $(n=13)$ at 1 -day post-injury, 30 min before the nociception assay. The panel $\mathbf{a}-\mathbf{b}$ show the average paw withdrawal threshold (PWT) or latency (PWL) of rats after DMET treatment (for PWT, lesion 
xtreatment interaction, $\mathrm{F}_{1,46}=4.184, p=0.0466$, for $\mathrm{PWL}$ lesion treatment interaction, $\mathrm{F}_{1,46}=8.795, p=0.0048$, post-hoc test: ${ }^{\star} p<0.05$, ${ }^{\star \star \star} p<0.001$ vs. saline, ${ }^{\wedge \wedge}{ }^{\wedge} p<0.001$ vs. DMET $5 \mu \mathrm{g}$, $\# \# \# p<0.001$ vs. DMET $10 \mu \mathrm{g}$ within group, $\& \& \& p<0.001$ vs. saline between groups by two-way ANOVA. Panel c-d summarizes the PWT and PWL of BPRA rats that received an intrathecal infusion of DMET alone or combined with the selective alpha-2A receptor antagonist, BLR-44408, but not the alpha-2C receptor antagonist JP-1302. All data are expressed as mean \pm SEM $* \star \star ~ p<0.001$ vs. BPRA+Veh group revealed by one-way ANOVA followed by Bonferroni post-hoc-test.

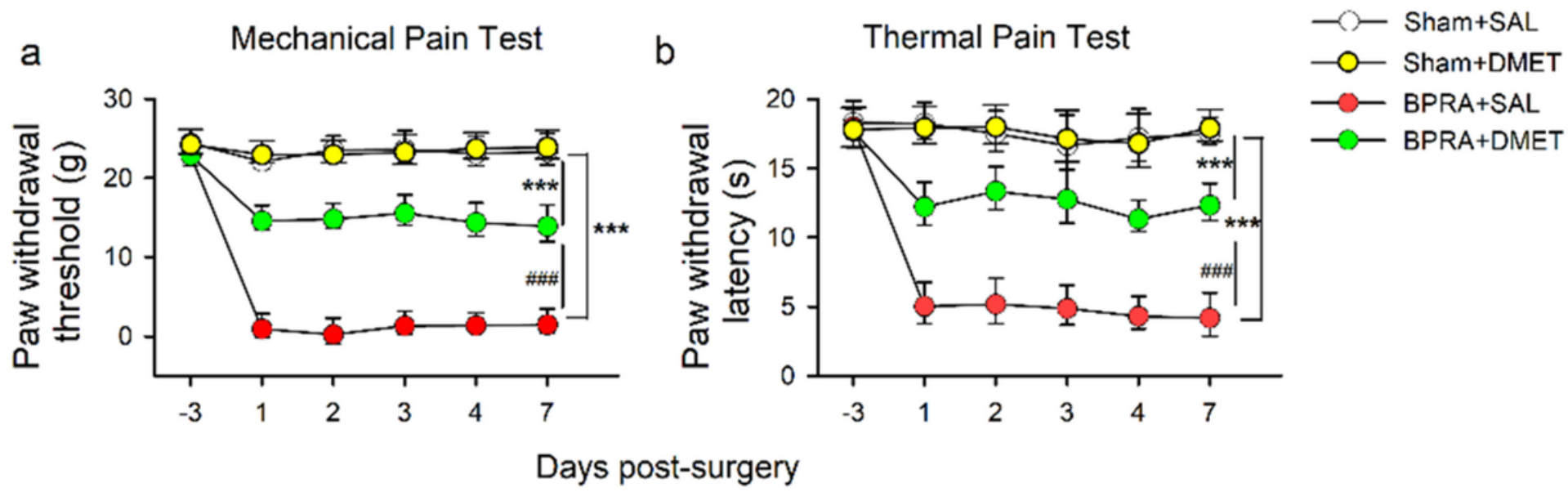

C
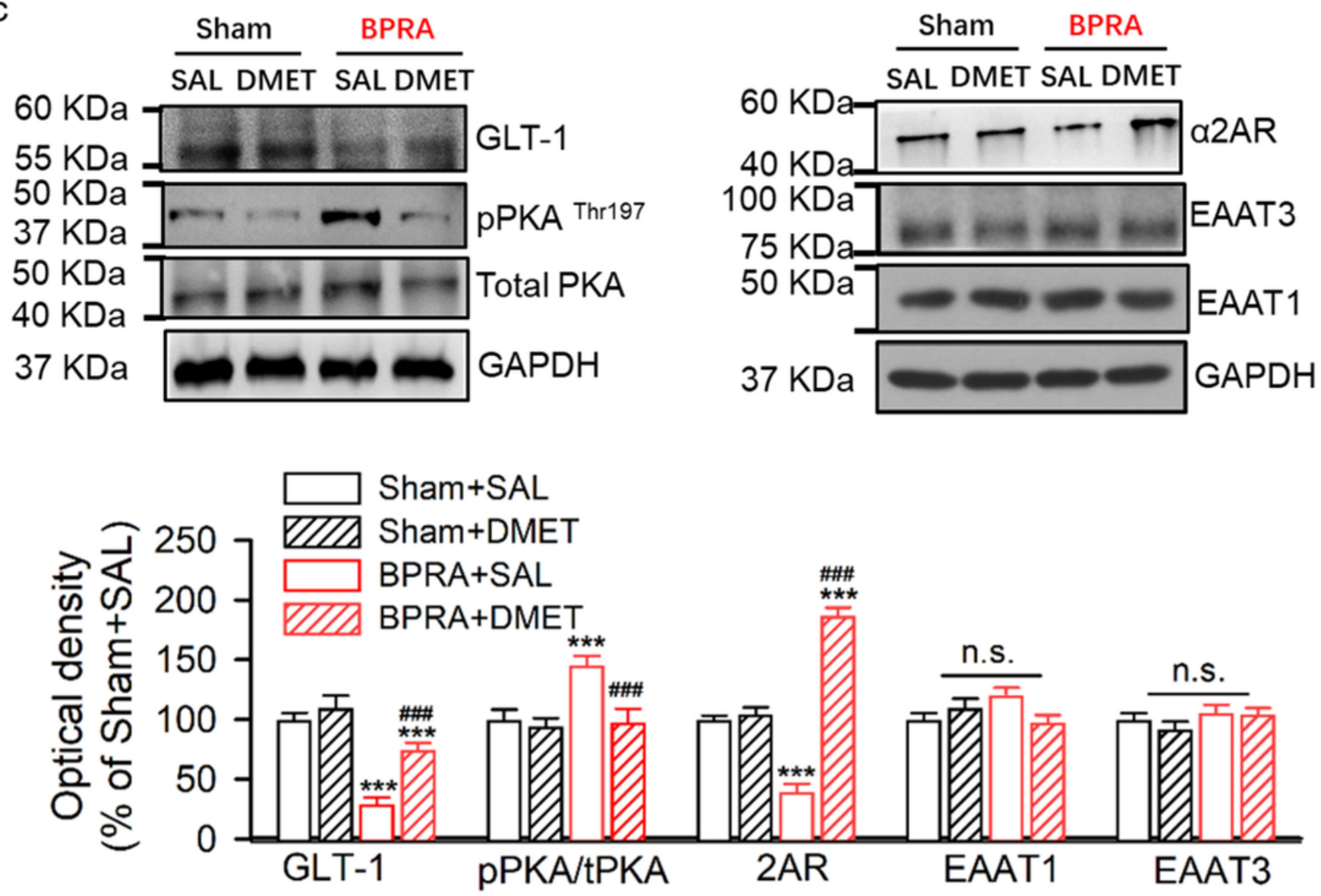

Figure 3 
Chronic intrathecal DMET treatment alleviates the brachial plexus roots avulsion induced pain and restored the reduced GLT-1 expression. Adult male SD rats that underwent C5-T1 BPRA injury or Sham lesion operation were randomly divided into intra-thecal DMET $(2 \mu \mathrm{g}, \mathrm{n}=10-13)$ or saline $(n=13)$ immediately after injury for three days and examined the mechanical and thermal pain assay during 7days post-injury (dpi). Panel a-b show the average paw withdrawal threshold (PWT) or latency (PWL) of rats after DMET treatment (for PWT, lesion $\times$ treatment interaction, $\mathrm{F}_{1,46}=4.184, p=0.0466$, for $\mathrm{PWL}$ lesion treatment interaction, $\mathrm{F}_{1,46}=8.795, p=0.0048$, post-hoc test: ${ }^{\star} p<0.05$, ${ }^{\star \star \star} p<0.001$ vs. saline, ${ }^{\wedge \wedge \wedge} p<0.001$ vs.

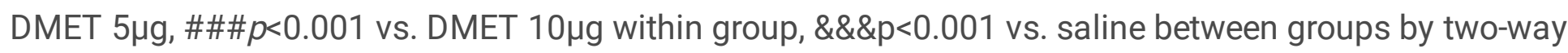
ANOVA. Panel c illustrates the protein level of alpha-2A receptor, phosphorylated PKA, GLAST (EAAT1), GLT-1(EAAT2), EAAC1(EAAT3) of the affected spinal cord of the above rats at $7 \mathrm{dpi}$. ${ }^{\star \star \star} p<0.001 \mathrm{vs.}$ BPRA+SAL within each protein subgroup, One-way ANOVA. 


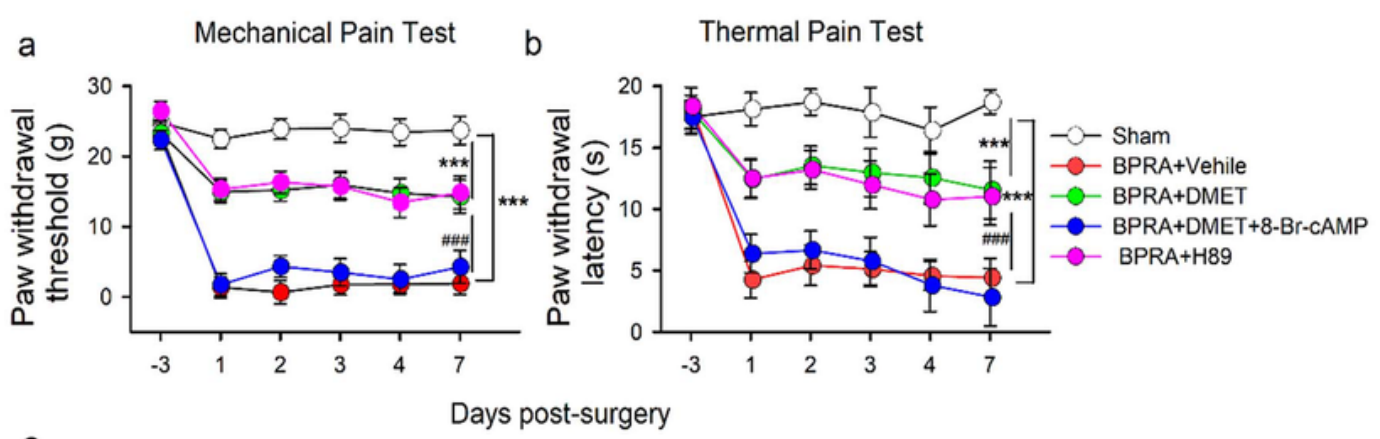

C

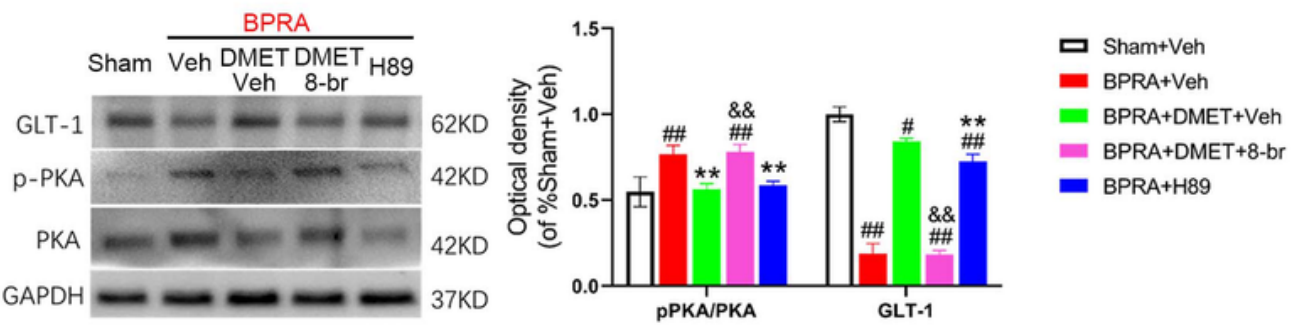

d
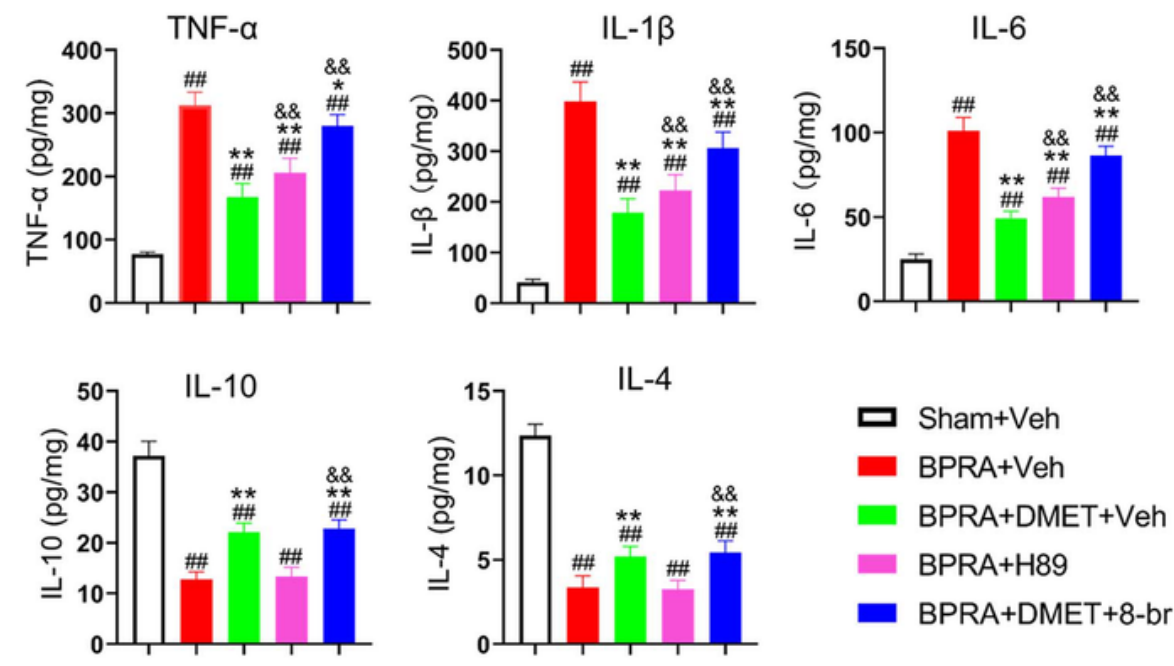

\section{Figure 4}

PKA involves DMET analgesic effect and spinal GLT-1 level restoration in BPRA rats. Adult male SD rats underwent C5-T1 BPRA injury or Sham lesion operation. The BPRA injured rats were randomly divided into four subgroups to evaluate pain assays 7 days post-injury (dpi), when received intrathecal administration of vehicle, DMET, DMET combined with PKA activator 8-Bromo-cAMP, or PKA selective inhibitor H89 alone, respectively, for three days. Panel a-b summarizes the change of average paw 
withdrawal threshold (PWT) or latency (PWL) of rats after various treatment strategies. ${ }^{\star \star} p<0.001$ vs. SHAM, \#\#\#p<0.001 vs. BPRA+DMET or H89, revealed by post-hoc test followed by two-way RM ANOVA. Panel $\mathbf{c}$ summarizes the related protein level change in the affected spinal cord of the above rats. Panel $\mathbf{d}$ listed anti-or pro cytokines level change of the injured spinal cord of the above rats. All data are expressed as mean \pm SEM $\# p<0.05, \# \# p<0.01$ vs. Sham $+V$ eh, ${ }^{* \star} p<0.01$ vs. BPRA $+V e h,{ }^{\& \&} p<0.01$ vs BPRA+DMET+Veh, revealed by Post-hoc test followed by One-way ANOVA.

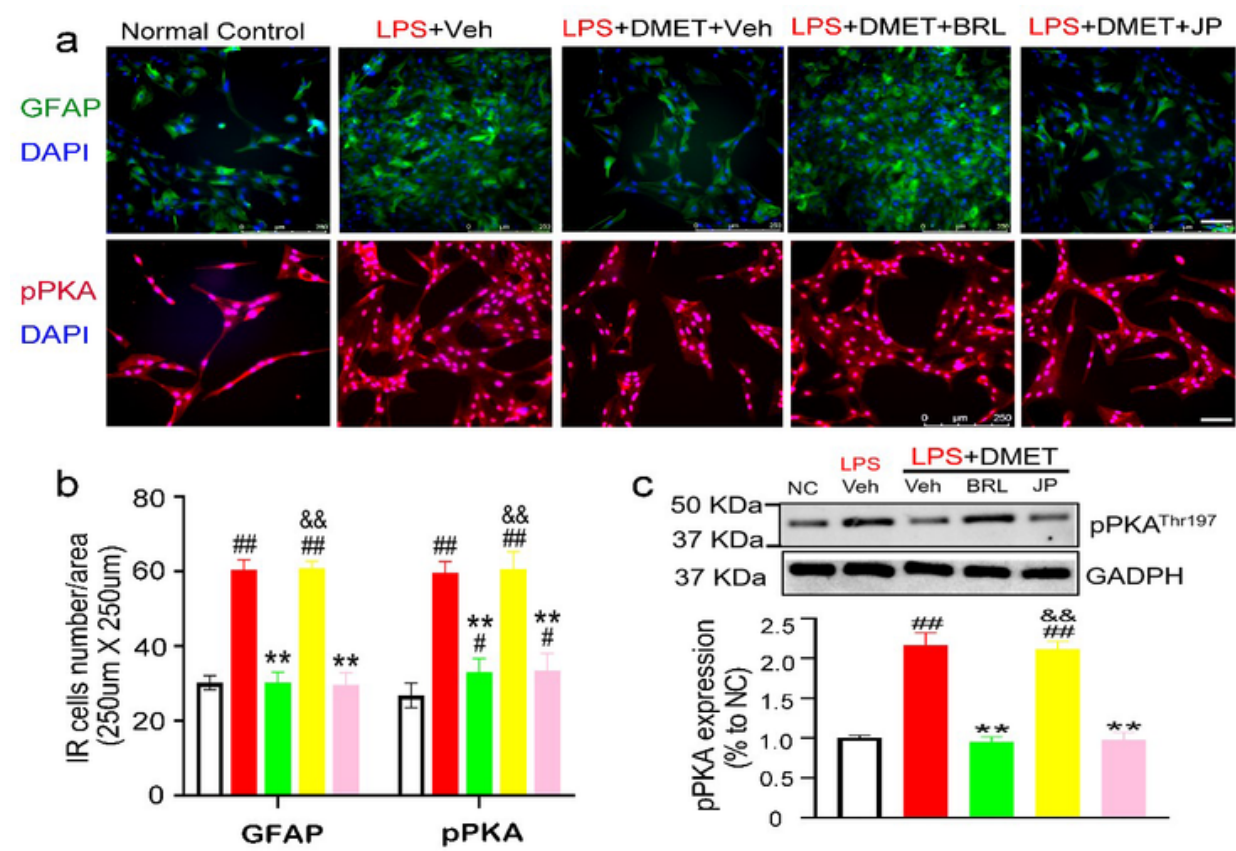

口 $\mathrm{NC}$ LPS LPS+DMET LPS+DMET+BRL-44408 LPS+DMET+JP-1302
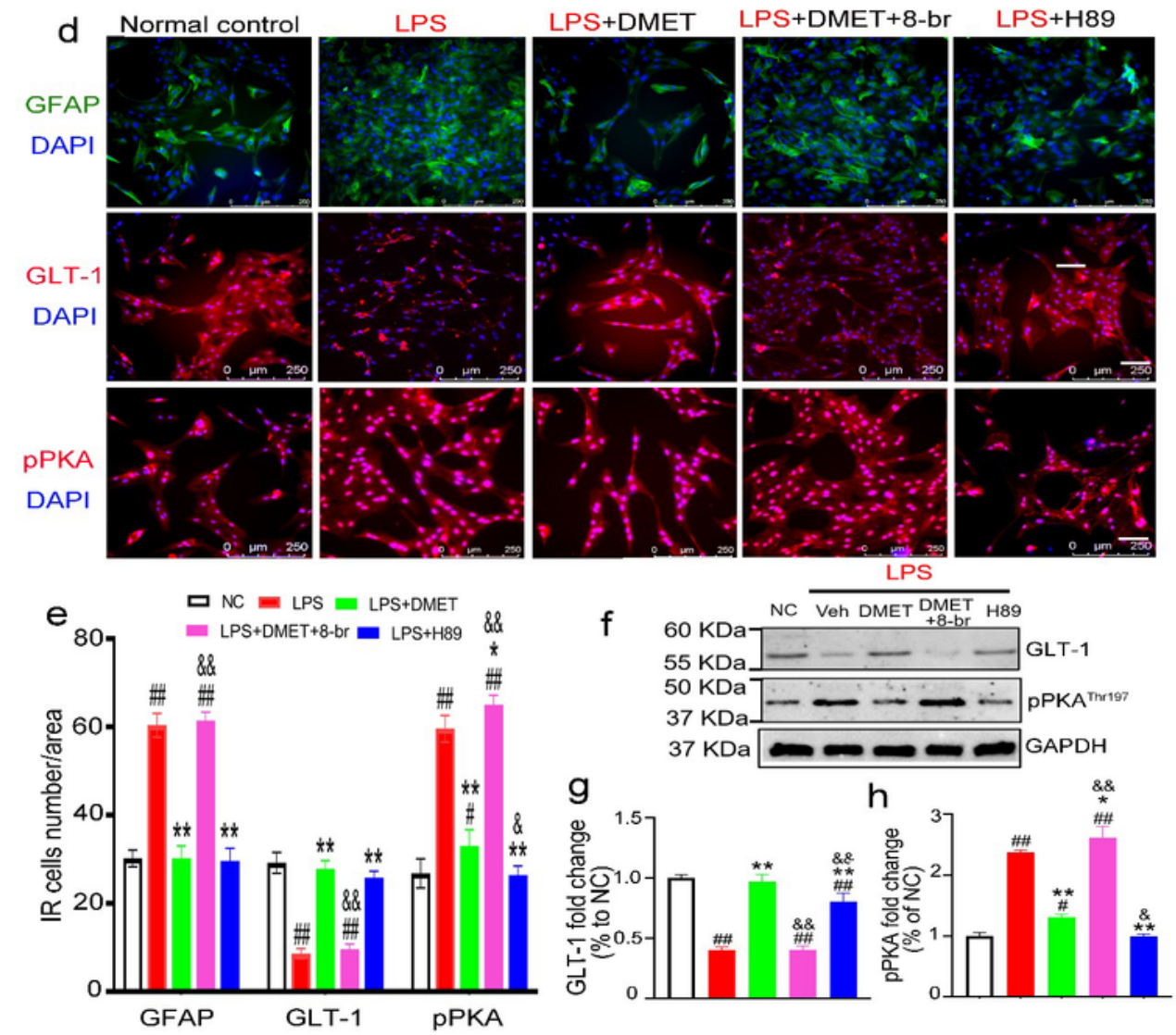


\section{Figure 5}

PKA signaling involves the GLT-1 restoration by DMET against LPS stimulation in Astrocytes. Panel a illustrated the representative microphotographs of GFAP (upper) and phosphorylated PKA (lower) immunostaining in astrocyte, counterstained by DAPI. The immunoreactive (IR) positive cells number were counted in the area $(250 \mu \mathrm{m} \times 250 \mu \mathrm{m})$ and summarized in panel $\mathbf{b}$. The mean value from cell counting was conducted on five selected areas per rat within each subgroup ( $n=3$ rats/subgroup). Panel $\mathbf{c}$ demonstrated the representative blotting bands (upper) and summarized them as a bar graph (lower). $\# p<0.05, \# \# p<0.01$ vs. Normal control (NC), ${ }^{*} p<0.01$ vs LPS+Veh, ${ }^{\&} \&<0.01$ vs LPS+DMET, revealed by Post-hoc test followed by One-way ANOVA. Panel $\mathbf{d}$ illustrates the representative microphotographs of GFAP (upper), GLT-1 (middle) phosphorylated PKA (lower) immunostaining in astrocyte, counterstained by DAPI. The IR cells number was summarized in panel $\mathbf{e}$. Panel $\mathbf{f}$ depicts the representative blotting bands, summarized in bar graphs $\mathbf{g}$ and $\mathbf{h}$. $\# p<0.05$, \#\#p<0.01 vs Normal control (NC), ${ }^{\star \star} p<0.01$ vs. LPS+Veh, ${ }^{\&} \&<0.01$ vs. LPS+DMET, revealed by Post-hoc test followed by One-way ANOVA.

\section{Supplementary Files}

This is a list of supplementary files associated with this preprint. Click to download.

- SupplementaryMaterial.pdf 